\title{
Motivation, Anxiety, and Emotional Intelligence Are Associated with the Practice of Contact and Non-Contact Sports: An Explanatory Model
}

\author{
Manuel Castro-Sánchez ${ }^{1}\left(\mathbb{D}\right.$, Amador J. Lara-Sánchez ${ }^{2}$, Félix Zurita-Ortega ${ }^{1}(\mathbb{D}$ and \\ Ramón Chacón-Cuberos ${ }^{3, *}$ (D) \\ 1 Department of Didactics of Musical, Plastic and Corporal Expression, University of Granada, \\ 18071 Granada, Spain \\ 2 Department of Didactics of Musical, Plastic and Corporal Expression, University of Jaén, 23071 Jaén, Spain \\ 3 Department of Research Methods and Diagnosis in Education, University of Granada, 18071 Granada, Spain \\ * Correspondence: rchacon@ugr.es; Tel.: +34-958-246-112
}

Received: 12 July 2019; Accepted: 5 August 2019; Published: 7 August 2019 updates

\begin{abstract}
Background: Several studies have shown that high anxiety impairs sport performance, making it important to develop strategies which improve the emotional self-regulation of athletes. The present study analyzed the relationship between emotional intelligence, motivational climate in sport, and anxiety according to participation in contact or non-contact sports, using multi-group structural equation modeling; (2) Methods: This research was conducted with a sample of 371 semi-professional athletes from Spain. The main variables were emotional intelligence, motivational climate in sport, and anxiety. A multi-group path analysis was developed and demonstrated acceptable fit $\left(\chi^{2}=418.60 ; \mathrm{df}=46 ; p<0.001\right.$; Comparative Fit Index $(\mathrm{CFI})=0.959$; Normalized Fit Index $(\mathrm{NFI})=0.955$; Incremental Fit Index $(\mathrm{IFI})=0.959$; Root Mean Square Error of Approximation (RMSEA) $=0.063)$; (3) Results: A negative association was identified between task-oriented climate and ego-oriented climate. Moreover, there was a positive relationship between task-oriented climate and emotional intelligence, which was strongest in individuals participating in non-contact sports. The relationship between ego-oriented climate and emotional intelligence was less evident. Furthermore, both state anxiety and trait anxiety were negatively correlated with emotional intelligence; (4) Conclusions: The key conclusion from the present research is that task-oriented motivational climates positively influence levels of emotional intelligence and anxiety, especially in contact sports. It is important to encourage the development of self-determined motivational climates in order to avoid emotional states which can hinder performance.
\end{abstract}

Keywords: Motivational Climate; Emotions; Stress; Contact Sports; Non-contact Sports

\section{Introduction}

Many studies of sport participation cite psychological variables as crucial tools for optimizing performance [1,2]. Previous research has shown that psychological preparation strategies can be successfully utilized to combat negative emotional states often experienced by athletes as anxiety, and to prolong high levels of adherence and performance throughout the sporting season [2]. A better understanding of the predictors of anxiety, motivational climate, and mental health enables efficacious strategies to be designed for various sport modalities [2-4]. The influence of the specific characteristics of various types of sports (including their internal dynamics, ways of association, or forms of contact between rivals and peers, etc.) on the aforementioned psychological variables, which will be further discussed here, have been previously discussed by Verner-Filion et al. [5]. 
Anxiety has been defined as an uncomfortable emotional state, brought about by stress when undertaking a task under pressure [6]. Although there is some research evidence affirming that anxiety can be a positive influence for athletes undertaking certain tasks [7], it is evident that anxiety generally exerts a negative influence on sport performance. For example, sports people who exhibit more successful sport performance usually demonstrate less anxiety during competition [8-10]. Further studies have demonstrated that strategies targeting working memory, perfectionism, or psychological skills can relieve anxiety [11,12]. It has also been demonstrated that athletes who possess self-determined motivations towards the practice of their sport present lower levels of state anxiety when confronted with negative situations [13]. The relationship between anxiety and sports motivation is therefore a pertinent topic.

Motivation is a key element of sport performance because it determines psychological aspects relating to decisions to engage, which ultimately influence the capacity to improve [14]. This research is based on self-determination theory [15], which describes the relationships between athletes' characteristics [16] and their goal orientations [17]. The present study considered the perceived motivational climates of athletes participating in contact and non-contact sports [18]. Previous research has identified that a task-oriented motivational climate, which emphasizes effort and goals which focus on personal improvement, encourages more successful sports performance than an ego-oriented climate, which focuses on successful outcomes and egocentric goals [19]. In sport contexts, the optimum climate will likely combine mostly task-oriented goals with some ego-oriented goals, in order to maintain competitive motivation whilst promoting important components of self-determined motivation, such as effort to improve or level of involvement $[15,18]$. In this context, athletes can maintain a level of anxiety that is productive to sport performance and effectively manage their emotions when sport performance does not bring about successful results, as has been shown by Castro-Sánchez et al. [20] in a sample of young footballers.

It is apparent that the motivational climate can have a marked impact on both sport performance and the health and wellbeing of the athlete [21]. A positive motivational climate can even improve personal satisfaction when competing $[22,23]$. This climate can be transmitted by the athlete's trainer, a team leader, or other close agents such as family or friends. When the trainer and athlete work in a positive motivational climate, group cohesion also improves [24]. Understanding and regulation of the emotions and moods of both trainer and athlete is linked to the motivational climate and essential for improving sport performance and well-being of the athlete [25].

Another important factor is emotional intelligence (EI). This construct describes the management and regulation of emotions and emotional information through behavioral patterns [26]. An athlete's ability to understand and regulate their emotions and those of others around them will profoundly influence their wellbeing, performance, and ability to socialize with others [27]. Previous research has reported higher scores for EI amongst more successful athletes relative to those with less success (Steca et al. [28]). Better capacity for emotional compression and emotional regulation has been shown to help to reduce levels of stress and anxiety during competitive periods [25], which has been related to lower neurotic states achieved by encouraging self-determined motivations in sport [29]. It is therefore important to analyze the relationship between these factors and identify strategies effective in producing environments which encourage productive outcomes for these constructs.

Engaging in sport of various types helps to improve EI [27]. While participation in both individual and team pursuits is efficacious in this respect [30], team pursuits seem to be more conducive to the positive development of aspects such as self-knowledge and self-control [31]. Good EI has been observed to regulate negative emotions during stressful situations, improving mental resilience and sports performance [32,33]. Engagement in certain sports may be more effective at developing EI than other sports [34]. For instance, athletes participating in individual versus collective sports, or those participating in contact versus non-contact sports, have different requirements regarding skills to identify, manage, and understand emotions [35]. Recognising these differences will enable specific athletes to conduct the most effective training by improving their emotional states [36]. 
Various studies have researched the effects of anxiety, EI, and motivation on performance $[37,38]$. More research is required to gain a greater understanding of the influence of these factors in the practice of different sports. Castro-Sánchez et al. [34] studied the different relationships between EI, anxiety, and motivational climate in athletes practicing individual sports and those practicing collective sports. The present study sought to expand upon this previous research by developing a structural model capable of examining the relationships of these variables within individuals practicing contact sports and individuals practicing non-contact sports.

Contact sports are undertaken in a common space in which peers and adversaries interact, and include combat sports and cooperation-opposition sports. Athletes participating in this type of sport therefore need to demonstrate particular physical and psychological characteristics which differ relative to non-contact sports, such as volleyball, tennis, or athletics [39]. Contact sports also require a greater capacity to pay attention to a greater number of stimuli. Further, overall performance success is more reliant upon the performance of other athletes (opponents as well as teammates), and so the ability to understand the emotions of rivals and companions is crucial [40]. In addition, contact sports carry a higher risk of injury, which can provoke situations of state anxiety [41]. Understanding the motivational orientations at play when practicing different types of sports and the motivational and emotional factors capable of regulating anxiety levels will enable athletes to maximize sports performance. Therefore, the promotion of certain types of motivational climates could help to improve anxiety levels and develop emotional intelligence in different sports contexts. In this way, their physical and mental state in the athlete could be favored, improving their well-being and favoring more adaptive and sustainable behaviors. The present study sought to answer the following research question: Are there differences in the relationships between motivational climate, anxiety, and emotional intelligence between athletes practicing contact sports and athletes practicing non-contact sports?

Given the findings of previous research, the following hypotheses were proposed:

Hypothesis 1 (H1). Task climate will be inversely related to state anxiety and this relationship will be more evident in participants of contact sports. In addition, ego climate will be positively related to state anxiety and this relationship will be more evident in non-contact sports.

Hypothesis 2 (H2). Emotional intelligence, ego climate, and both types of anxiety will be inversely related, with these relationships being more evident in contact sports. In addition, emotional intelligence will be positively associated with task-oriented climate in both contact and non-contact sports.

The aims of the present research were therefore: to analyze the relationships between emotional intelligence, anxiety, and motivational climate in athletes participating in contact and non-contact sports using multi-group structural equations.

\section{Materials and Methods}

\subsection{Subjects and Design}

A descriptive, cross-sectional study using self-report measures was conducted with a sample of 371 semi-professional athletes from Spain. The gender distribution was $63.2 \%$ male and $36.8 \%$ female and participants ranged from 18 to 27 years old $(M=21.19$ years; $S D=3.11)$. With regards to contact sports, the sample included 141 footballers and 18 taekwondo practitioners. With regards to non-contact sports, the sample included 172 athletes who practiced athletics and 41 paddle tennis players. Participants were recruited using convenience sampling from various sports clubs: 8 athletics clubs, 12 football clubs, 2 paddle tennis clubs, and 2 taekwondo clubs. Individual monitoring ensured data were not duplicated. The method of maximum likelihood in the analysis of covariance was employed, and valid coefficients were obtained for the root mean square error of approximation; further, the standard error bias for all parameters did not exceed 5\% [42]. 


\subsection{Measures}

Motivational climate in sport was assessed using the Perceived Motivational Climate in Sports Questionnaire (PMCSQ-2), which has been validly translated into Spanish by González-Cutre et al. [43]. The measure is composed of 33 items rated on a five point Likert scale ranging from " $1=$ totally disagree," to " $5=$ totally agree." The questionnaire establishes two dimensions: task-oriented climate and ego-oriented climate. Each dimension is measured by three subscales: effort/improvement, cooperative learning, and important role describe the task dimension, whereas unequal recognition, punishment for mistakes, and intra-team rivalry describe the ego dimension. This instrument demonstrated good reliability, with a Cronbach's alpha of $\alpha=0.88$ for the ego dimension and $\alpha=0.92$ for the task dimension.

Emotional intelligence in sport was assessed using the Schutte Self Report Inventory (SSRI) [44], which has been adapted into Spanish by García-Coll et al. [45]. This scale establishes four dimensions: emotional perception, emotional self-regulation, interpersonal-emotional management, and the use of emotions. The scale comprises 33 items which evaluate the respondents' capacity to recognize, understand, and control one's own emotions and those of other people. Items were rated on a five point Likert scale ranging from " $1=$ totally disagree," to " $5=$ totally agree." We employed the shortened 30 item form proposed by García-Coll et al. [45], which eliminates the negatively framed items 5, 28, and 33 in order to improve the viability of the analysis. This instrument demonstrated good internal consistency, with a Cronbach's alpha coefficient of $\alpha=0.92$. Reliability values for each dimension were as follows: hetero-emotional management $(\alpha=0.92)$, self-emotional management $(\alpha=0.94)$, emotion perception $(\alpha=0.89)$, and emotion utilization $(\alpha=0.81)$.

Anxiety was assessed using the State-Trait Anxiety Inventory (STAI) (Spielberger et al. [46]). This scale has been employed widely worldwide, particularly in the context of health and sport [47,48]. Responses are provided on a four point Likert scale ranging from " $0=$ nothing" to " $3=$ a lot." The scale comprises 40 items which are divided in two dimensions: trait anxiety (T-Anxiety) and state anxiety (S-Anxiety). Categories of anxiety were designated according to specifications of the original version of the scale [46]. This instrument demonstrated a Cronbach's alpha of $\alpha=0.94$. The S-anxiety subscale produced a reliability score $\alpha=0.91$, and the T-anxiety subscale reliability score was $\alpha=0.89$.

Type of sport practiced was assessed using an ad-hoc questionnaire. Sports were categorized into contact sports (i.e., football or combat sports) and non-contact sports (i.e., athletics or racket sports) for entry into the multi-group structural equation analysis.

\subsection{Procedure}

Questionnaires were distributed between September and December of 2017 and they were completed by athletes prior to training. Of the data collected, 56 questionnaires were discarded due to being incompletely or incorrectly completed. The study conformed to the guidelines of the Helsinki Declaration (World Medical Association) concerning research projects (Law 223/2004 of 6 February), in addition to national legislation on clinical trials (relating to biomedicine Law 14/2007 of 3 July) and in compliance with participant confidentiality law (Law 15/1999 of 13 December). In addition, this research obtained ethics permission from the Ethics Committee of the University of Granada (462/CEIH/2017).

\subsection{Statistical Analysis}

IBM SPSS@version 22.0 for Windows was used for the basic descriptive analysis. The IBM AMOS $囚 23$ software was employed to analyze the associations between the relevant variables of the structural equation modeling. First, a path model was constructed establishing the relationships between constructs. Second, a multi-group analysis was conducted, grouping participants by entering type of sport practice as an independent variable $(1=$ contact sports-footballers and taekwondo practitioners; 2 = non-contact sports—athletics practitioners and paddle tennis players). This structural 
model allowed associations between variables to be differentiated according to contact/non-contact sport participation. The maximum likelihood (ML) method was used to estimate relationships between variables. This method was chosen because it is consistent, unbiased, and invariant to types of scale when variables are normally distributed.

Path diagrams present 12 observable variables and 3 latent variables. The observed relationships between indicators were explanations for the latent variables. Measurement error was directly controlled through observable variables. Unidirectional arrows show the direction of influence between observable and latent variables, and these also provide the regression coefficients. In addition, bidirectional arrows show the associations between latent variables (Figure 1).

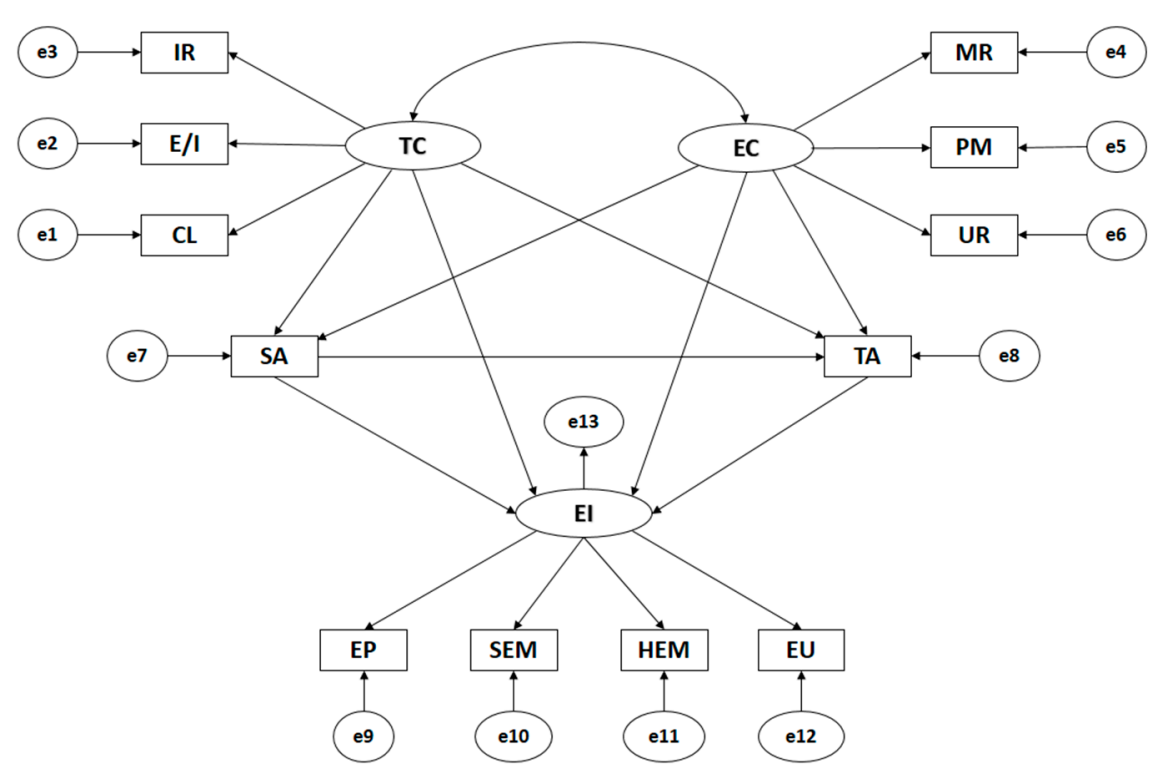

Figure 1. Theoretical model for the relationships between the variables. Note 1: TC, task-oriented climate; CL, cooperative learning; E/I, effort/improvement; IR, important role; EC, ego-oriented climate; MR, rivalry between members; $P M$, punishment of mistakes; UR, unequal recognition; SA, state anxiety; TA, trait anxiety; EI, emotional intelligence; HEM, hetero-emotional management; SEM, emotional self-management; EP, emotion perception; EU, emotion utilization.

Task-oriented climate (TC) and ego-oriented climate (EC) represent the exogenous variables and were each inferred by three indicators which described the three subscales of each dimension. TC was indicated by the subscales effort/improvement (EI), important role (IR), and cooperative learning (CL). EC was indicated by the subscales unequal recognition (UR), punishment of mistakes (PM), and rivalry between (MR). General emotional intelligence (EI) was the endogenous variable influenced by trait anxiety (TA), state anxiety (SA), ego-oriented climate (EC), and task-oriented climate (TC).

Model fit was tested to verify compatibility with the empirical data. Goodness of fit evaluation was conducted [49]. Chi-squared analysis was conducted when non-significant p-values indicated good model fit. Comparative fit index (CFI), normalized fit index (NFI), and incremental fit index (IFI) values higher than 0.90 indicate acceptable model fit, while values higher than 0.95 indicate excellent model fit. Root mean square error of approximation (RMSEA) values below 0.08 indicate acceptable model fit, while values below 0.05 indicate excellent model fit.

\section{Results}

\subsection{Measurement Model}

The global structural equation model developed for the relationships between motivational climate, anxiety, and emotional intelligence in athletes demonstrated good fit indices. The chi-squared value was significant $\left(\chi^{2}=418.60 ; \mathrm{df}=46 ; p<0.001\right)$. Despite this, values could not be interpreted 
in the standard manner as it was highly influenced by sample size [42]. Thus, other standardized fit indexes were used that are less sensitive to sample size. CFI, NFI, and IFI produced excellent values of $0.959,0.955$, and 0.959 , while RMSEA was acceptable at 0.063 .

Multi-group analysis distinguishing between participants of contact and non-contact sports also demonstrated acceptable fit indices, though reliability was lower than for the global model (Table 1 ). Chi-squared analysis produced a significant outcome for contact sports $\left(\chi^{2}=617.93, \mathrm{df}=92, p<0.001\right)$ and non-contact sports $\left(\chi^{2}=598.47, \mathrm{df}=86, p<0.001\right)$. For contact sports, the remaining standardized fit indexes were acceptable: $\mathrm{CFI}=0.944, \mathrm{NFI}=0.935, \mathrm{IFI}=0.944$, and RMSEA $=0.072$. For non-contact sports, these indices were also acceptable: $\mathrm{CFI}=0.947, \mathrm{NFI}=0.939, \mathrm{IFI}=0.945$, and RMSEA $=0.067$.

Table 1. Measurement model.

\begin{tabular}{cccc}
\hline INDICES & Global Model & Model 1 (Contact Sports) & Model 2 (Non-Contact Sports) \\
\hline$\chi^{2}$ & 418.60 & 617.93 & 598.47 \\
Df & 46 & 92 & 86 \\
$p$ & $p<0.001$ & $p<0.001$ & $p<0.001$ \\
CFI & 0.959 & 0.944 & 0.947 \\
NFI & 0.955 & 0.935 & 0.939 \\
IFI & 0.959 & 0.944 & 0.945 \\
RMSEA & 0.063 & 0.072 & 0.067 \\
\hline
\end{tabular}

Note 1: Df, degrees of freedom; CFI, comparative fit index; NFI, normalized fit index; IFI, incremental fit index; RMSEA, root mean square error of approximation.

\subsection{Global Structural Model}

The regression weights and standard regression weights for the global structural model are shown in both Table 2 and Figure 2. Regression weights should significantly differ from zero, with negative values being undesirable. A positive association was identified between all indicators of motivational climate and its two dimensions $(p<0.005)$. In addition, a negative relationship between task-oriented climate and ego-oriented climate $(p=0.005 ; \mathrm{r}=-0.215)$ was identified. Further, direct relationships $(p<0.005)$ were observed between each indicator of emotional intelligence and its global dimension.

A significant difference existed between the indicators for all latent variables $(p<0.005)$, with all variables also being directly associated. The indicator describing effort/improvement was most strongly associated $(r=0.904)$ with a task-oriented climate, with cooperative learning $(r=0.908)$ and important role $(r=0.884)$ also showing strong associations. With regards to ego climate, the strongest relationship was identified with the indicator describing unequal recognition $(r=0.919)$, with punishment of mistakes $(r=0.853)$ and rivalry between members $(r=0.666)$ also showing strong associations. With regards to emotional intelligence, the strongest relationship was identified with the indicator describing emotion perception $(r=0.906)$. The indicator describing use of emotion $(r=0.674)$ demonstrated the weakest relationship.

A task-oriented climate was negatively associated with both state anxiety $(p<0.005 ; \mathrm{r}=-0.221)$ and trait anxiety $(\mathrm{r}=0.056)$. An ego-oriented climate was positively associated with state anxiety $(p<0.005 ; r=0.154)$, but was not significantly associated with trait anxiety. State and trait anxiety were directly and positively associated $(\mathrm{r}=0.809 ; p<0.005)$.

Finally, emotional intelligence was positively associated with both a task-oriented climate $(p<0.005 ; \mathrm{r}=0.429)$ and an ego-oriented climate $(p<0.01 ; \mathrm{r}=0.090)$. Emotional intelligence was negatively associated with trait anxiety $(\mathrm{r}=-0.305 ; p<0.005)$, but was not significantly associated with state anxiety. 
Table 2. Regression weights for the global structural model.

\begin{tabular}{|c|c|c|c|c|c|c|c|}
\hline \multicolumn{3}{|c|}{ Relationships } & \multicolumn{4}{|c|}{ Regression Weights } & \multirow{3}{*}{$\begin{array}{c}\text { Standard Regression Weights } \\
\text { Estimates } \\
0.154\end{array}$} \\
\hline & & & \multirow{2}{*}{$\begin{array}{c}\text { Estimates } \\
3.412\end{array}$} & \multirow{2}{*}{$\begin{array}{l}\text { Error } \\
0.557\end{array}$} & \multirow{2}{*}{$\begin{array}{c}\text { Critical Ratio } \\
4.870\end{array}$} & \multirow{2}{*}{$\begin{array}{c}\mathbf{P} \\
* * *\end{array}$} & \\
\hline SA & $\leftarrow$ & $\mathrm{EC}$ & & & & & \\
\hline SA & $\leftarrow$ & $\mathrm{TC}$ & -2.493 & 0.471 & -7.173 & $* * *$ & -0.221 \\
\hline $\mathrm{TA}$ & $\leftarrow$ & $\mathrm{TC}$ & 1.276 & 0.289 & 2.855 & $* *$ & 0.056 \\
\hline $\mathrm{TA}$ & $\leftarrow$ & SA & 0.702 & 0.018 & 42.724 & $* * *$ & 0.809 \\
\hline $\mathrm{TA}$ & $\leftarrow$ & $\mathrm{EC}$ & 0.355 & 0.334 & 0.681 & 0.496 & 0.013 \\
\hline EI & $\leftarrow$ & SA & 0.002 & 0.002 & -0.419 & 0.675 & -0.019 \\
\hline EI & $\leftarrow$ & $\mathrm{TA}$ & -0.012 & 0.002 & -6.882 & $* * *$ & -0.305 \\
\hline EI & $\leftarrow$ & $\mathrm{TC}$ & 0.298 & 0.020 & 14.167 & $* * *$ & 0.429 \\
\hline EI & $\leftarrow$ & $\mathrm{EC}$ & 0.032 & 0.023 & 3.052 & $* *$ & 0.090 \\
\hline $\mathrm{E} / \mathrm{I}$ & $\leftarrow$ & $\mathrm{TC}$ & 0.892 & 0.019 & 45.131 & $* * *$ & 0.904 \\
\hline IR & $\leftarrow$ & $\mathrm{TC}$ & 1.049 & 0.024 & 43.338 & $* * *$ & 0.884 \\
\hline MR & $\leftarrow$ & $\mathrm{EC}$ & 1.000 & - & - & - & 0.666 \\
\hline UR & $\leftarrow$ & $\mathrm{EC}$ & 1.236 & 0.056 & 23.989 & $* * *$ & 0.919 \\
\hline PM & $\leftarrow$ & EC & 0.980 & 0.047 & 24.109 & $* * *$ & 0.853 \\
\hline CL & $\leftarrow$ & $\mathrm{TC}$ & 1.000 & - & - & - & 0.908 \\
\hline HEM & $\leftarrow$ & EI & 0.906 & 0.023 & 40.288 & $* * *$ & 0.861 \\
\hline SEM & $\leftarrow$ & EI & 0.956 & 0.022 & 42.040 & $* * *$ & 0.881 \\
\hline EP & $\leftarrow$ & EI & 1.000 & - & - & - & 0.906 \\
\hline UE & $\leftarrow$ & EI & 0.810 & 0.030 & 26.462 & $* * *$ & 0.674 \\
\hline EC & $\leftrightarrow$ & $\mathrm{TC}$ & -0.096 & 0.015 & -6.259 & $* * *$ & -0.215 \\
\hline
\end{tabular}

Note 1: TC, task-oriented climate; CL, cooperative learning; E/I, effort/improvement; IR, important role; EC, ego-oriented climate; MR, rivalry between members; PM, punishment of mistakes; UR, unequal recognition; SA, state anxiety; TA, trait anxiety; EI, emotional intelligence; HEM, hetero-emotional management; SEM, emotional self-management; EP, emotion perception; EU, emotion utilization. Note 2: *** statistically significant relationship at level $p=0.005 ;{ }^{* *}$ statistically significant relationship at level $p=0.01$; ${ }^{*}$ statistically significant relationship at level $p=0.05$.

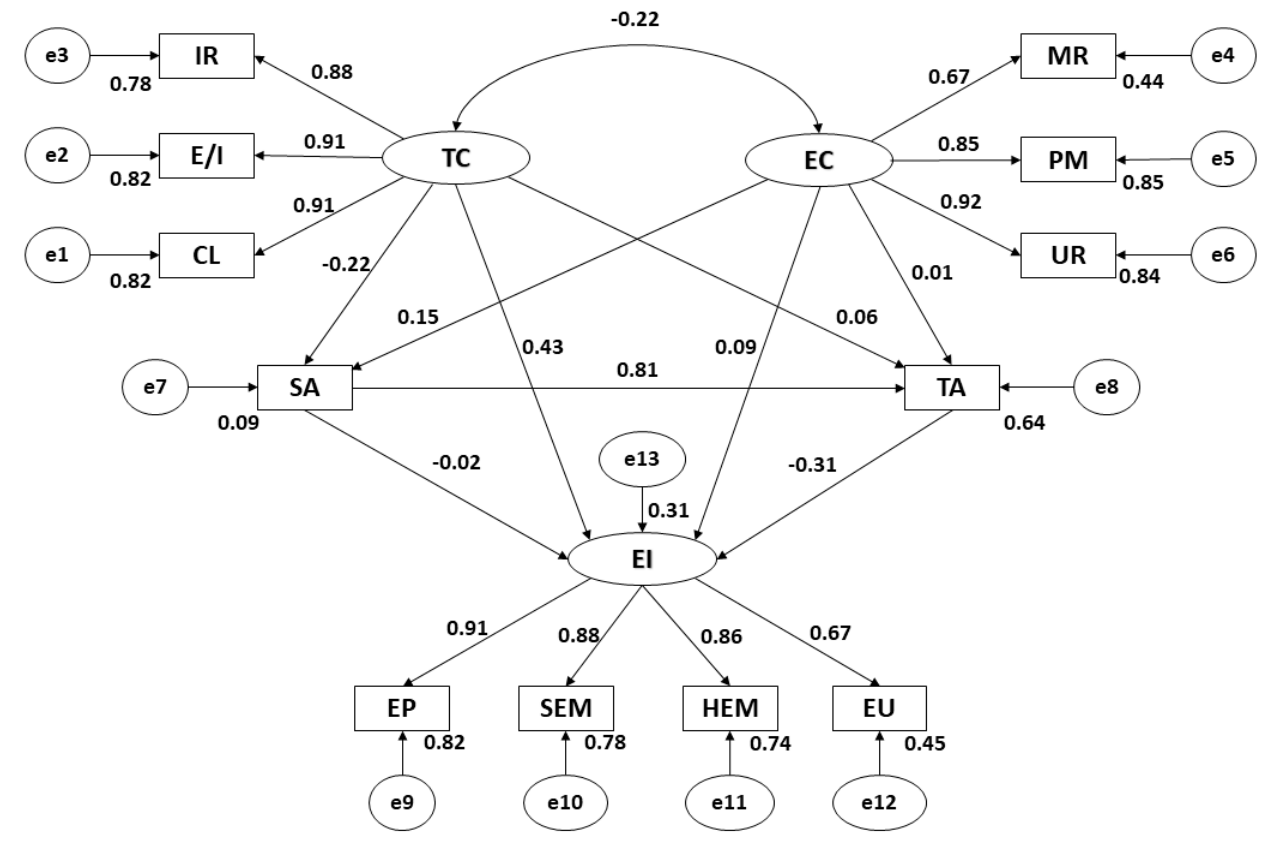

Figure 2. Global structural equation model for all subjects. Note 1: TC, task-oriented climate; CL, cooperative learning; E/I, effort/improvement; IR, important role; EC, ego-oriented climate; MR, rivalry between members; PM, punishment of mistakes; UR, unequal recognition; SA, state anxiety; TA, trait anxiety; EI, emotional intelligence; HEM, hetero-emotional management; SEM, emotional self-management; EP, emotion perception; EU, emotion utilization. 


\subsection{Multigroup Analysis: Relationships between Variables for Contact Sports}

The estimated values of the model parameters for non-contact sports are shown in both Table 3 and Figure 3. Regression weights should significantly differ from zero, with negative variables being undesirable. Positive relationships were identified between all indicators of motivational climate and its two dimensions $(p<0.005)$. In addition, a negative relationship existed between a task-oriented climate and an ego-oriented climate $(p=0.005 ; \mathrm{r}=-0.208)$. Positive relationships $(p<0.005)$ were also observed between all indicators of emotional intelligence and their respective global dimensions.

Indicators of all latent variables were significantly different $(p<0.005)$, with all variables being directly associated. A task-oriented climate was most strongly associated with the indicator describing effort/improvement $(\mathrm{r}=0.915)$, with indicators describing cooperative learning $(\mathrm{r}=0.904)$ and important role $(r=0.885)$ also being strongly associated. An ego-oriented climate was most strongly correlated with the indicator describing unequal recognition $(\mathrm{r}=0.928)$, with indicators describing punishment of errors $(r=0.845)$ and rivalry between members $(r=0.748)$ also being strongly associated. Emotional intelligence was most strongly associated with emotion perception $(r=0.900)$ and least strongly associated with use of emotion $(r=0.664)$.

A negative and significant relationship was observed between a task-oriented climate and state anxiety $(p<0.005 ; \mathrm{r}=-0.160)$, whereas a task-oriented climate was positively correlated with trait anxiety $(r=0.091)$. With regards to an ego-oriented climate, a positive relationship was observed with state anxiety $(p<0.005 ; \mathrm{r}=0.203)$ and a no significant relationship was observed with trait anxiety. Finally, state anxiety and trait anxiety were directly associated $(r=0.782 ; p<0.005)$.

Emotional intelligence was positively associated with a task-oriented climate $(\mathrm{r}=0.483 ; p<0.005)$ but was not significantly correlated with an ego-oriented climate. Finally, global levels of emotional intelligence were positively associated with trait anxiety $(\mathrm{r}=-0.272 ; p<0.005)$ but were not associated with state anxiety.

Table 3. Regression weights for non-contact sports.

\begin{tabular}{|c|c|c|c|c|c|c|c|}
\hline \multicolumn{3}{|c|}{ Relationships } & \multicolumn{4}{|c|}{ Regression Weights } & \multirow{3}{*}{$\begin{array}{c}\text { Standard Regression Weights } \\
\text { Estimates } \\
0.203\end{array}$} \\
\hline & & & \multirow{2}{*}{$\begin{array}{c}\text { Estimates } \\
3.412\end{array}$} & \multirow{2}{*}{$\begin{array}{l}\text { Error } \\
0.699\end{array}$} & \multirow{2}{*}{$\begin{array}{c}\text { Critical Ratio } \\
4.878\end{array}$} & \multirow{2}{*}{$\begin{array}{c}\mathbf{P} \\
* * *\end{array}$} & \\
\hline SA & $\leftarrow$ & EC & & & & & \\
\hline SA & $\leftarrow$ & $\mathrm{TC}$ & -2.493 & 0.634 & -3.930 & $* * *$ & -0.160 \\
\hline $\mathrm{TA}$ & $\leftarrow$ & $\mathrm{TC}$ & 1.276 & 0.380 & 3.357 & $* * *$ & 0.091 \\
\hline $\mathrm{TA}$ & $\leftarrow$ & SA & 0.702 & 0.024 & 29.775 & $* * *$ & 0.782 \\
\hline TA & $\leftarrow$ & $\mathrm{EC}$ & 0.355 & 0.417 & 0.851 & 0.395 & 0.024 \\
\hline EI & $\leftarrow$ & SA & 0.002 & 0.002 & 0.909 & 0.363 & 0.053 \\
\hline EI & $\leftarrow$ & TA & -0.012 & 0.003 & -4.764 & $* * *$ & -0.272 \\
\hline EI & $\leftarrow$ & $\mathrm{TC}$ & 0.298 & 0.025 & 11.807 & $* * *$ & 0.483 \\
\hline EI & $\leftarrow$ & EC & 0.032 & 0.026 & 1.213 & 0.225 & 0.048 \\
\hline $\mathrm{E} / \mathrm{I}$ & $\leftarrow$ & $\mathrm{TC}$ & 0.892 & 0.026 & 34.895 & $* * *$ & 0.915 \\
\hline IR & $\leftarrow$ & $\mathrm{TC}$ & 1.049 & 0.032 & 32.802 & $* * *$ & 0.885 \\
\hline MR & $\leftarrow$ & $\mathrm{EC}$ & 1.000 & - & - & - & 0.748 \\
\hline UR & $\leftarrow$ & $\mathrm{EC}$ & 1.236 & 0.056 & 22.076 & $* * *$ & 0.928 \\
\hline PM & $\leftarrow$ & $\mathrm{EC}$ & 0.980 & 0.046 & 21.509 & $* * *$ & 0.845 \\
\hline CL & $\leftarrow$ & $\mathrm{TC}$ & 1.000 & - & - & - & 0.904 \\
\hline HEM & $\leftarrow$ & EI & 0.906 & 0.031 & 28.867 & $* * *$ & 0.849 \\
\hline SEM & $\leftarrow$ & EI & 0.956 & 0.032 & 30.110 & $* * *$ & 0.870 \\
\hline EP & $\leftarrow$ & EI & 1.000 & - & - & - & 0.900 \\
\hline UE & $\leftarrow$ & EI & 0.810 & 0.042 & 19.335 & $* * *$ & 0.664 \\
\hline EC & $\leftrightarrow$ & TC & -0.108 & 0.023 & -4.660 & $* * *$ & -0.208 \\
\hline
\end{tabular}

Note 1: TC, task-oriented climate; CL, cooperative learning; E/I, effort/improvement; IR, important role; EC, ego-oriented climate; MR, rivalry between members; PM, punishment of mistakes; UR, unequal recognition; SA, state anxiety; TA, trait anxiety; EI, emotional intelligence; HEM, hetero-emotional management; SEM, emotional self-management; EP, emotion perception; EU, emotion utilization. Note $2:{ }^{* * *}$ statistically significant relationship at level $p=0.005 ;{ }^{* *}$ statistically significant relationship at level $p=0.01 ;{ }^{*}$ statistically significant relationship at level $p=0.05$. 


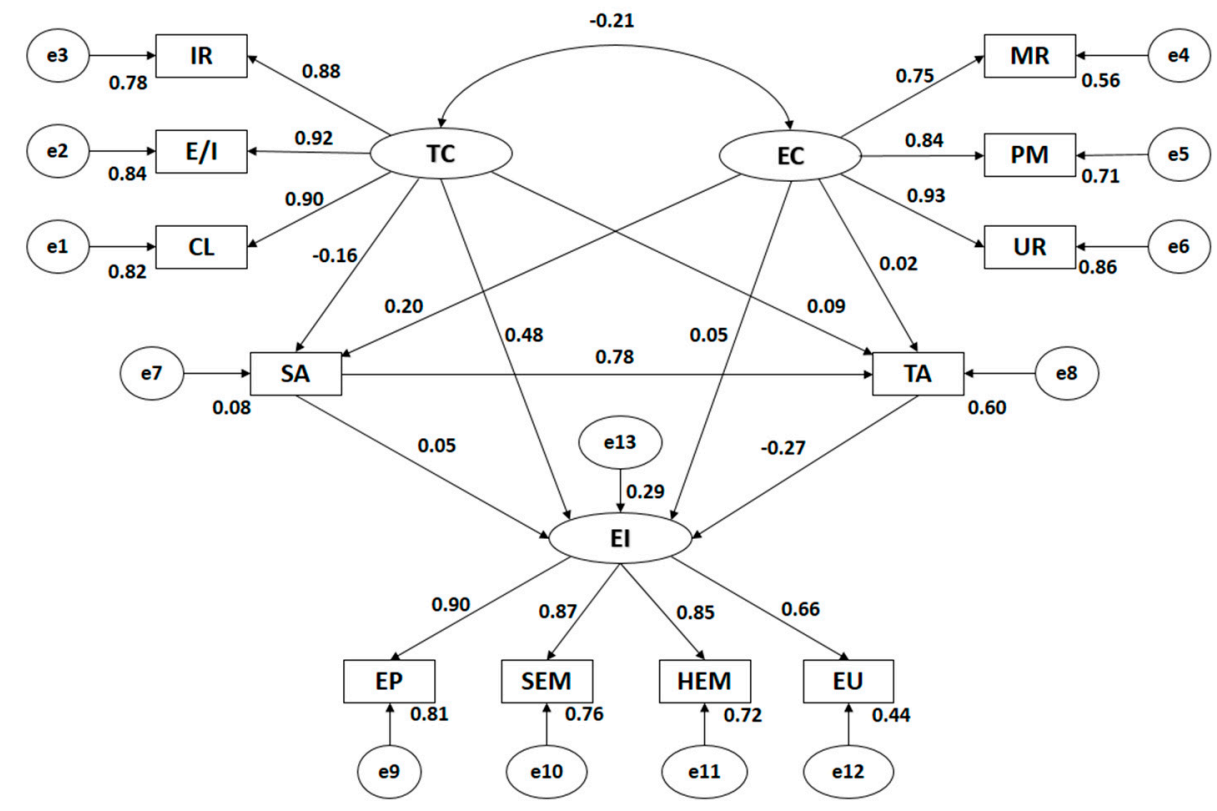

Figure 3. Structural equation model for non-contact sports. Note 1: TC, task-oriented climate; CL, cooperative learning; E/I, effort/improvement; IR, important role; EC, ego-oriented climate; MR, rivalry between members; PM, punishment of mistakes; UR, unequal recognition; SA, state anxiety; TA, trait anxiety; EI, emotional intelligence; HEM, hetero-emotional management; SEM, emotional self-management; EP, emotion perception; EU, emotion utilization.

\subsection{Multigroup Analysis: Relationships between Variables for Non-Contact Sports}

The estimated values of the model parameters for non-contact sports are shown in both Table 4 and Figure 4. Significant and direct relationships were observed between all categories of motivational climate and its dimensions $(p<0.005)$. Task-oriented climate was negatively correlated with ego-oriented climate $(p<0.005 ; \mathrm{r}=-0.208)$. All indicators of emotional intelligence were significantly and positively associated $(p<0.005)$.

The indicators of each latent variable were significantly and positively correlated $(p<0.005)$. A task-oriented climate was most strongly associated with the indicator describing cooperative learning $(r=0.915)$, with indicators describing effort/improvement $(r=0.889)$ and important role $(r=0.882)$ also being strongly related. An ego-oriented climate was most strongly associated with the indicator describing unequal recognition $(r=0.901)$, with indicators describing punishment of errors $(r=0.867)$ and rivalry between members $(r=0.541)$ also being strongly related. Emotional intelligence was most strongly associated with the indicator describing emotion perception $(r=0.909)$ and least associated with the indicator describing use of emotion $(r=0.695)$.

A task-oriented climate was negatively associated with state anxiety $(p<0.005 ; \mathrm{r}=-0.321)$, but did not demonstrate any relationship with trait anxiety. State anxiety and trait anxiety were directly associated $(p<0.005 ; \mathrm{r}=0.845)$.

Emotional intelligence was positively associated with both a task-oriented climate $(p<0.005$; $r=0.320)$ and an ego-oriented climate $(p<0.005 ; r=0.124)$. In addition, emotional intelligence was negatively associated with both trait anxiety $(p<0.005 ; \mathrm{r}=-0.283)$ and state anxiety $(\mathrm{r}=-0.186$; $p=0.012$ ). 
Table 4. Regression weights for contact sports.

\begin{tabular}{cccccccc}
\hline \multirow{2}{*}{ Relationships } & \multicolumn{5}{c}{ Regression Weights } & & Standard Regression Weights \\
\cline { 3 - 7 } & & & Estimations & Error & Critical Ratio & $\mathbf{P}$ & Estimations \\
\hline SA & $\leftarrow$ & EC & 2.249 & 0.954 & 2.357 & $*$ & 0.113 \\
SA & $\leftarrow$ & TC & -4.696 & 0.679 & -6.919 & $* * *$ & -0.321 \\
TA & $\leftarrow$ & TC & 0.387 & 0.428 & 0.905 & 0.365 & 0.025 \\
TA & $\leftarrow$ & SA & 0.884 & 0.028 & 31.759 & $* * *$ & 0.845 \\
TA & $\leftarrow$ & EC & 0.632 & 0.570 & 1.110 & 0.267 & 0.030 \\
EI & $\leftarrow$ & SA & -0.009 & 0.004 & -2.518 & $*$ & -0.186 \\
EI & $\leftarrow$ & TA & -0.014 & 0.004 & -3.916 & $* * *$ & -0.283 \\
EI & $\leftarrow$ & TC & 0.239 & 0.033 & 7.140 & $* * *$ & 0.320 \\
EI & $\leftarrow$ & EC & 0.126 & 0.045 & 2.831 & $* *$ & 0.124 \\
E/I & $\leftarrow$ & TC & 0.832 & 0.029 & 28.687 & $* * *$ & 0.889 \\
IR & $\leftarrow$ & TC & 0.993 & 0.035 & 28.250 & $* * *$ & 0.882 \\
MR & $\leftarrow$ & EC & 1.000 & - & - & - & 0.901 \\
UR & $\leftarrow$ & EC & 1.616 & 0.137 & 11.784 & $* * *$ & 0.867 \\
PM & $\leftarrow$ & EC & 1.502 & 0.126 & 11.943 & $* * *$ & 0.915 \\
CL & $\leftarrow$ & TC & 1.000 & - & - & - & 0.874 \\
HEM & $\leftarrow$ & EI & 0.957 & 0.034 & 27.958 & $* * *$ & 0.901 \\
SEM & $\leftarrow$ & EI & 0.863 & 0.029 & 29.759 & $* * *$ & 0.909 \\
EP & $\leftarrow$ & EI & 1.000 & - & - & - & 0.695 \\
UE & $\leftarrow$ & EI & 0.773 & 0.042 & 18.364 & $* * *$ & -0.223 \\
EC & $\leftrightarrow$ & TC & -0.074 & 0.018 & -4.103 & $* * *$ & \\
\hline
\end{tabular}

Note 1: TC, task-oriented climate; CL, cooperative learning; E/I, effort/improvement; IR, important role; EC, ego-oriented climate; MR, rivalry between members; PM, punishment of mistakes; UR, unequal recognition; SA, state anxiety; TA, trait anxiety; EI, emotional intelligence; HEM, hetero-emotional management; SEM, emotional self-management; EP, emotion perception; EU, emotion utilization. Note 2: *** statistically significant relationship at level $p=0.005 ;{ }^{* *}$ statistically significant relationship at level $p=0.01$; ${ }^{*}$ statistically significant relationship at level $p=0.05$.

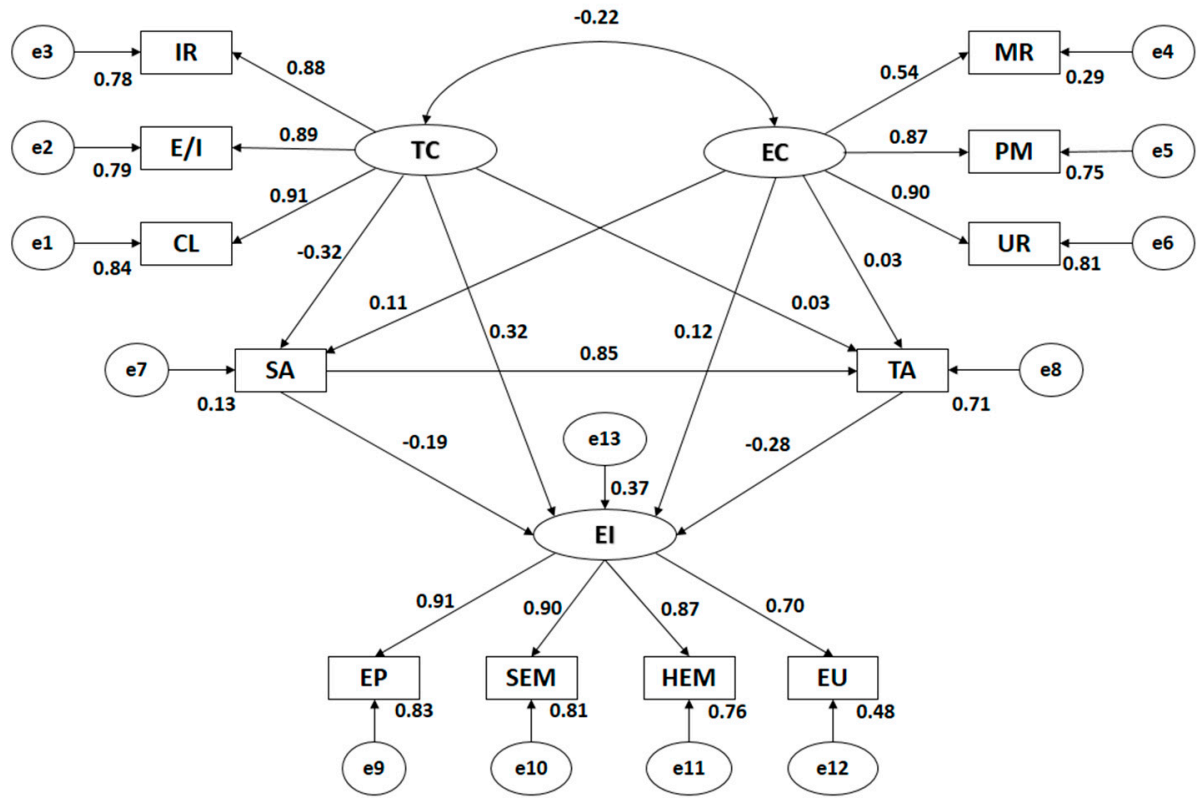

Figure 4. Structural Equation Model for contact sports. Note 1: TC, task-oriented climate; CL, cooperative learning; E/I, effort/improvement; IR, important role; EC, ego-oriented climate; MR, rivalry between members; PM, punishment of mistakes; UR, unequal recognition; SA, state anxiety; TA, trait anxiety; EI, emotional intelligence; HEM, hetero-emotional management; SEM, emotional self-management; EP, emotion perception; EU, emotion utilization.

\section{Discussion}

The aim of this study was to analyze the relationships between emotional intelligence, motivational climate, and anxiety in two sports modalities, namely, contact and non-contact sports, using multi-group 
structural equation modelling. The path models developed demonstrated acceptable fit indices, suggesting that they validly explained the associations between the measured variables in this sample of Spanish semi-professional athletes, developing previous international and national work [50-54]. The global model revealed excellent fit indices, which was anticipated as a greater number of participants were included than in the multi-group analysis. Nevertheless, standardized regression weights were acceptably large for all variables included in the structural models of the multi-group analysis for both contact and non-contact sports. This was due to the greater specificity afforded by this type of analysis.

Firstly, a negative association was identified between a task-oriented climate and an ego-oriented climate. With regards to non-contact sports, the indicator with the greatest relationship with a task-oriented climate was effort/improvement. With regards to contact sports, cooperative learning was the indicator most correlated with a task-oriented climate. These findings suggest that participants of non-contact sports tend to focus more on personal effort, given that they have no contact with the adversary and achieving a successful outcome depends largely on their own technical and physical abilities [55]. On the other hand, contact sports (such as opposition-cooperation sports) typically involve greater interaction with peers and rivals, which explains why cooperative learning would be of greater relevance $[55,56]$. Given these results, it can be concluded that knowledge of personal progress is important in order to achieve and maintain high levels of effort. A recommendation would be to conduct training sessions centred on tasks which allow improvements in the mastery of certain skills, to be routinely checked and subsequent feedback provided to inform the correction of technical imperfections. Another effective strategy is the setting of goals which allow the athlete to progress realistically, avoid frustration, and reach a higher level of satisfaction when participating in sport. Further, in order to promote cooperative learning, activities should be proposed in which joint goals are achieved through collaboration and cooperation amongst all members of the group, rather than tasks which promote the singular achievement of personal goals [57].

Cooperative learning is a key factor influencing contact sports, as participants must attend to a larger number of variables influencing both personal performance and overall success, making self-regulation of emotions particularly important [58,59]. In both contact and non-contact sports, unequal recognition was the indicator which was most strongly associated with an ego climate. This demonstrates that the trainer's opinion carries great weight with all types of athletes, who strive to obtain better sport performance than their competitors and even companions. The reaction of their trainer to these attempts is one of the most influential factors affecting the degree of the athletes' engagement in the sport $[60,61]$. In order to avoid extrinsic motivations, which develop in contexts with ego-oriented motivational climates, situations which encourage athletes to make external comparisons should be avoided or minimized. Such contexts can lead to demotivation or an excessive dependence on extrinsic motivations, which are linked to reward seeking and damaging levels of anxiety [62].

Emotional intelligence was most strongly associated with emotional perception and least associated with use of emotion. This was the case for both contact and non-contact sports. The perception of emotion was then followed by the capacity to regulate and control emotions, with the latter being more difficult to master [44]. The association between task-oriented climate and emotional intelligence was strongest in non-contact sports, while ego-oriented climate and emotional intelligence were only related in contact sports. A comprehensive training program targeting all indicators of emotional intelligence must form part of the emotional preparation of athletes. Given the value of these dimensions, trainers should implement intervention programs that equip athletes to manage and use emotions productively. Effective strategies could include discussion groups and role-play activities, in which individuals learn to recognize and use others' emotions through situations of introspection [63].

Findings regarding EI may be partly explained by the observation that EI allows for the understanding that certain emotions produced by a particular motivation are more favourable for performance [64]. The relationship with task climate may be more pronounced in non-contact sports as participants place greater focus on achievement and personal effort, whereas participants in contact sports orient more towards overcoming the opponent through direct contact $[65,66]$. Nonetheless, 
the positive relationship between an ego-oriented motivational climate and EI in contact sports was surprising. This may be partly explained by the fact that contact sport participants are more accustomed to managing negative emotions linked to competition, such as rivalry, envy, or feelings of losing. They may therefore have a better understanding of how to achieve successful emotional management $[67,68]$. Given the apparent benefits of high levels of emotional intelligence on sports performance, intervention programs should be introduced to reduce the fear of failure, increase focus on important and relevant stimuli, enable physical and mental relaxation and dispositional flow, and increase the confidence of the athlete [69].

In both contact and non-contact sports, a direct and positive relationship was found between state anxiety and trait anxiety, with this relationship being stronger in contact sports. It is well established that athletes with anxious personalities increase their levels of state anxiety when confronted with stress factors, while those with low-anxiety traits are better able to control their anxiety levels $[70,71]$. The relationship between state and trait anxiety may be more prevalent in contact sports than in non-contact sports, as performance success depends on the competitor's actions in addition to the individual's, and the actions of others cannot be easily predicted or controlled [72]. Physiological techniques, such as Jacobson muscle relaxation, respiratory control techniques, inoculation of stress, biofeedback, systematic desensitization, or the programming of pre-execution routines, should be used to reduce the stress and anxiety experienced by athletes. Strategies used to implant a task-oriented motivational climate, such as cognitive techniques including Schultz's autogenic training, control of thoughts, visualization, or cognitive restructuring, are also recommended [73].

A task-oriented climate was negatively associated with state anxiety in participants of both sports modalities, although the relationship was more pronounced in contact sports. Conversely, a positive and direct relationship was observed between a task-oriented climate and trait anxiety in participants of non-contact sports, whereas no relationship was observed in participants of contact sports. As previously discussed, task-oriented motivations are typically accompanied by a lowering of anxiety levels in athletes. This is because task-oriented participants are more engaged in the process of participation rather than the outcome, which in turn can result in greater ability to exert control over the outcome. The opposite is true with regards to extrinsic motivation [74]. Given this relationship between motivational climate and anxiety, athletes should be offered sessions focused on the promotion of task-oriented climates which target reduced levels of anxiety. To this end, a potentially useful strategy is the TARGET technique. TARGET is a psychological intervention which is based on the six factors of task, authority, recognition, group, evaluation, and time. In summary, activities are prepared which offer athletes personal challenge, opportunities to be actively involved, and opportunities to make active decisions. These activities are then introduced into training and even competition. Moreover, it is essential to regularly recognize the individual and collective effort and achievement of athletes, encourage teamwork in a pleasant environment, and use correct evaluation techniques adjusted to the time requirements and characteristics of the individual or group [75].

With regards to EI, negative relations were identified with trait anxiety in both contact and non-contact sport participants. In contact sports, EI was only correlated with state anxiety, and this relationship was also in a negative direction. This corroborates findings from studies of individual and collective sports. Ros et al. [76] identified that emotional intelligence allows athletes to adopt an orientation towards the task, increasing their focus on personal improvement, enabling them to reduce their anxiety levels [77]. Moreover, higher emotional intelligence enables participants to better understand the stressors which produce a negative emotional state, thus enabling them to avoid these stressors and avoid high state anxiety [76]. Given the relationship between EI and anxiety, sports psychologists should introduce to their athletes coping strategies linked to the control of emotions and solving of problems. Emotional coping strategies involve actively rethinking and mentally restructuring each stressful situation in order to adopt techniques which are effective for the individual (e.g., decreased attention, distancing, rejection, mental dissociation, or increased positive desires). Rather than attempt to solve the problem that is causing anxiety, these techniques seek to regulate 
the emotional response to that problem in order to avoid harm to the participant. In order to have a positive effect, these techniques begin with a planning phase and end with expert support in putting the newly planned coping skills into practice [68].

Finally, it is important to identify the limitations of the present study. Firstly, relatively few contact sport participants were recruited and included in the sample. In addition, the sample size in general may have prevented firmer conclusions from being made. Secondly, the study employed a cross-sectional design, which precludes causal conclusions from being made. Finally, it is essential to highlight that the structural equation model performed did not consider other variables that may influence the findings obtained, such as sex or age. However, the objectives of the study were linked to the knowledge of the relationships between anxiety, motivation, and emotional intelligence according to the sport practiced, considering that these do not vary greatly according to sex. Future studies should aim to develop intervention programs which reduce stress and anxiety in sports participants. Given that this variable is negatively correlated with a task-oriented motivational climate and high emotional intelligence, training of athletes should include exercises based on maximising effort for personal improvement, cooperation, emotional perception and emotional regulation, and adverse situations. A deeper understanding of the relationships identified in the present study would be enabled by collecting data at various times throughout the sporting season in order to elucidate differences between pre-competitive and competitive periods, or differences following positive and negative performance outcomes.

\section{Conclusions}

The present study identified a positive relationship between a task-oriented climate and emotional intelligence, in addition to a negative relationship between these variables and anxiety. These associations can be differentiated according to the type of sport practiced (contact sports and non-contact sports). Here, we address the study hypotheses:

- Hypothesis 1 (H1) was supported, as the relationship between a task-oriented climate and anxiety was both negative and more pronounced amongst contact sport participants. In addition, an ego-oriented climate was positively associated with state anxiety, and this relationship was more pronounced in non-contact sport participants.

- Hypothesis 2 (H2) was partially supported. Emotional intelligence was negatively associated with both state and trait anxiety, and this relationship was more pronounced in contact sport participants. The relationship between emotional intelligence and an ego-oriented climate was positive for both contact and non-contact sport participants.

The key conclusion of the present research was the negative association between an ego-oriented climate and a task-oriented climate. Moreover, it was found that a task-oriented climate was most strongly associated with effort/improvement, and cooperative learning in non-contact and contact sport participants, respectively. With regards to an ego-oriented climate, unequal recognition had the greatest influence in both sports modalities. The aspects of emotional intelligence had a greater influence in contact sports than non-contact sports. Moreover, there was a positive relationship between a task-oriented climate and emotional intelligence, which was most prevalent in non-contact sports. The relationship between an ego-oriented climate and emotional intelligence was less pronounced. Furthermore, there was a negative relationship between state and trait anxiety and emotional intelligence. Finally, the strongest indicator of emotional intelligence was emotional perception, with use of emotion being the weakest indicator. In conclusion, it is clearly important to promote self-determined motivations towards sport participation in order to improve emotional intelligence and reduce levels of anxiety, particularly in non-contact sports. Therefore, the promotion of certain types of motivational climates could help improve physical and mental states in athletes, favoring their well-being and more adaptive and sustainable behaviors. 
Author Contributions: M.C.-S. and R.C.-C. conceived the hypothesis of this study. M.C.-S., F.Z.-O. and A.L.-S. participated in data collection. M.C.-S., R.C.-C. and F.Z.-O. analyzed the data. All authors contributed to data interpretation of statistical analysis. M.C.-S., A.L.-S. and R.C.-C. wrote the paper. All authors read and approved the final manuscript.

Funding: This research received no external funding.

Conflicts of Interest: The authors declare no conflict of interest.

\section{References}

1. Hanton, S.; Mellalieu, S.; Williams, J. Undestanding and Managing Stress in Sport; McGraw-Hill: New York, NY, USA, 2015.

2. Musculus, L.; Lobinger, B. Psychological Characteristics in Talented Soccer Players-Recommendations on How to Improve Coaches' Assessment. Front. Psychol. 2018, 9. [CrossRef] [PubMed]

3. Hauw, D. Enaction and intervention in sports psychology among elite athletes and those in training. Can. J. Behav. Sci. 2018, 50, 54-64. [CrossRef]

4. Thelwell, R.C.; Wood, J.; Harwood, C.; Woolway, T.; Van Raalte, J.L. The role, benefits and selection of sport psychology consultants: Perceptions of youth-sport coaches and parents. Psychol. Sport Exerc. 2018, 35, 131-142. [CrossRef]

5. Verner-Filion, J.; Vallerand, R.J.; Donahue, E.G.; Moreau, E.; Martin, A.; Mageau, G.A.; Martin, A. Passion, coping, and anxiety in sport: The interplay between key motivational and self-regulatory processes. Int. J. Sport Psychol. 2014, 45, 516-537.

6. Cheng, W.; Hardy, L.; Markland, D. Toward a three-dimensional conceptualization of performance anxiety: Rationale and initial measurement development. Psychol. Sport Exerc. 2009, 10, 271-278. [CrossRef]

7. Ngo, V.; Richards, H.; Kondric, M. A multidisciplinary investigation of the effects of competitive state anxiety on serve kinematics in table tennis. J. Hum. Kinet. 2017, 55, 83-95. [CrossRef] [PubMed]

8. Di Rienzo, F.; Martinent, G.; Leveque, L.; Maclntyre, T.; Collet, C.; Guillot, A. The influence of gate start position on physical performance and anxiety perception in expert BMX athletes. J. Sports Sci. 2018, 36, 311-318. [CrossRef] [PubMed]

9. Ford, J.; Ildefonso, K.; Jones, M.; Arvinen-Barrow, M. Sport-related anxiety: Current insights. J. Sports Med. 2017, 8, 205-212. [CrossRef]

10. Spindler, D.; Allen, M.; Vella, S.; Swann, C. The psychology of elite cycling: A systematic review. J. Sports Sci. 2018, 36, 1943-1954. [CrossRef] [PubMed]

11. Capranica, L.; Condello, G.; Tornello, F.; Iona, T.; Chiodo, S.; Valenzano, A.; De Rosas, M.; Messina, G.; Tessitore, A.; Cibelli, G. Salivary alpha-amylase, salivary cortisol, and anxiety during a youth taekwondo championship. An observational study. Medicine 2017, 96, e7272. [CrossRef]

12. Ducrocq, E.; Wilson, M.; Smith, T.; Derakshan, N. Adaptive Working Memory Training Reduces the Negative Impact of Anxiety on Competitive Motor Performance. J. Sport Exerc. Psychol. 2017, 39, 412-422. [CrossRef] [PubMed]

13. Gunnell, K.E.; Crocker, P.R.; Mack, D.E.; Wilson, P.M.; Zumbo, B.D. Goal contents, motivation, psychological need satisfaction, well-being and physical activity: A test of self-determination theory over 6 months. Psychol. Sport Exerc. 2014, 15, 19-29. [CrossRef]

14. Sheehan, R.; Herring, M.; Campbell, M. Associations Between Motivation and Mental Health in Sport: A Test of the Hierarchical Model of Intrinsic and Extrinsic Motivation. Front. Psychol. 2018, 9. [CrossRef] [PubMed]

15. Deci, E.L.; Ryan, R.M. Intrinsic Motivation and Self-Determination in Human Behavior; Plenum: New York, NY, USA, 1985.

16. Li, C.; Kee, Y.; Wang, X.; Guo, Q. The Big Five Personality Traits and physical and emotional exhaustion among athletes: The mediating role of autonomous and controlled motivation. Int. J. Sport Psychol. 2018, 49, 1-16. [CrossRef]

17. Nicholls, J. The Competitive Ethos and Democratic Education; Harvard University Press: Cambridge, UK, 1989.

18. Ames, C. Achievement Goals, Motivational Climate, and Motivational Processes; Human Kinetics: Champaign, IL, USA, 1992.

19. Eys, M.; Jewitt, E.; Evans, M.; Wolf, S.; Bruner, M.; Loughead, T. Coach-initiated motivational climate and cohesion in youth sport. Res. Quarter. Exerc. Sport 2013, 84, 373-383. [CrossRef] [PubMed] 
20. Castro-Sánchez, C.; Zurita-Ortega, F.; Chacón-Cuberos, R.; Padial-Ruz, R.; Martínez-Martínez, A. Levels of anxiety in players of the lower categories. Sport TK 2018, 7, 53-60.

21. Gustafsson, H.; Carlin, M.; Podlog, L.; Stenling, A.; Lindwall, M. Motivational profiles and burnout in elite athletes: A person-centered approach. Psychol. Sport Exerc. 2018, 35, 118-125. [CrossRef]

22. Fransen, K.; Boen, F.; Vansteenkiste, M.; Metens, N.; Vande, G. The power of competence support: The impact of coaches and athlete leaders on intrinsic motivation and performance. Scan. J. Med. Sci. Sports 2018, 28, 725-745. [CrossRef]

23. Kolavis, H.; Sari, I.; Celik, N. Parent-initiated motivational climate and self-determined motivation in youth sport: How should parents behave to keep their child in sport? Kinesiology 2017, 49, 217-224. [CrossRef]

24. Heuze, J.; Eys, M.; Dubuc, M.; Bosselut, G.; Couture, R. Cohesion, psychological needs, and intrinsic motivation in youth team sport contexts. Int. J. Sport Psychol. 2018, 49, 55-73.

25. Lee, Y.H.; Chelladurai, P. Emotional intelligence, emotional labor, coach burnout, job satisfaction, and turnover intention in sport leadership. Eur. Sport Manag. Q. 2018, 18, 393-412. [CrossRef]

26. Petrices, K. Trait emotional intelligence theory. Ind. Organ. Psychol. 2010, 3, 136-139. [CrossRef]

27. Laborde, S.; Guilien, F.; Watson, M. Trait emotional intelligence questionnaire full-form and short-form versions: Links with sport participation frequency and duration and type of sport practiced. Personal. Individ. Differ. 2017, 108, 5-9. [CrossRef]

28. Steca, P.; Baretta, D.; Greco, A.; D’Addario, M.; Monzani, D. Associations between personality, sports participation and athletic success. A comparison of Big Five in sporting and non-sporting adults. Personal. Individ. Differ. 2018, 121, 176-183. [CrossRef]

29. Perry, J.; Ross, M.; Weinstock, J.; Gfeller, J. Examining the Interrelationships between Motivation, Conscientiousness, and Individual Endurance Sport Performance. J. Sports Sci. 2017, 5, 146-156. [CrossRef]

30. Laborde, S.; Guillén, F.; Mosley, E. Positive personality-trait-like individual differences in athletes from individual-and team sports and in non-athletes. Psychol. Sport Exerc. 2016, 26, 9-13. [CrossRef]

31. Akelaitis, A.; Malinauskas, R. The expression of emotional skills among individual and team sports male athletes. Ped. Psychol. Med.-Biol. Probl. Phys. Tranining Sports 2018, 22, 62-67. [CrossRef]

32. Cowden, R.G.; Fuller, D.K.; Anshel, M.H. Psychological predictors of mental toughness in elite tennis: An exploratory study in learned resourcefulness and competitive trait anxiety. Percept. Mot. Skills 2014, 119, 661-678. [CrossRef] [PubMed]

33. O'Connor, P.; Nguyen, J.; Anglim, J. Effectively Coping With Task Stress: A Study of the Validity of the Trait Emotional IntelligenceQuestionnaire-Short Form (TEIQue-SF). J. Personal. Assess. 2017, 99, 304-314. [CrossRef]

34. Castro-Sánchez, M.; Zurita-Ortega, F.; Chacón-Cuberos, R.; López-Gutiérrez, C.J.; Zafra-Santos, E. Emotional Intelligence, Motivational Climate and Levels of Anxiety in Athletes from Different Categories of Sports: Analysis through Structural Equations. Int. J. Environ. Res. Public Health 2018, 15, 894. [CrossRef]

35. Keeler, L.A. The Differences in Sport Aggression, Life Aggression, and Life Assertion Among Adult Male and Female Collision, Contact, and Non-Contact Sport Athletes. J. Sport Behav. 2007, 30, 1-21.

36. Mann, D.T.; Williams, A.M.; Ward, P.; Janelle, C.M. Perceptual-cognitive expertise in sport: A meta-analysis. J. Sport Exerc. Psychol. 2017, 29, 457-478. [CrossRef]

37. Olmedilla, A.; García, C.; Martínez, F. Psychological factors and vulnerability to sports injuries in soccer players. Rev. Psicol. Dep. 2006, 15, 37-52.

38. Hodgson, L.; Butt, J.; Maynard, I. Exploring the psychological attributes underpinning elite sports coaching. Int. J. Sports Sci. Coach. 2017, 12, 439-451. [CrossRef]

39. Campo, M.; Laborde, S.; Mosley, E. Emotional Intelligence Training in Team Sports the Influence of a Season Long Intervention Program on Trait Emotional Intelligence. J. Individ. Differ. 2016, 37, 152-158. [CrossRef]

40. Grao-Cruces, A.; Fernández-Martínez, A.; Teva-Villén, M.R.; Nuviala, A. Physical self-concept and intention to be physically activie in participants of the sport schools program. J. Sport Health Res. 2017, 9, 15-26.

41. Hagan, J.; Pollmann, D.; Schack, T. Interaction between Gender and Skill on Competitive State Anxiety Using the Time-to-Event Paradigm: What Roles Do Intensity, Direction, and Frequency Dimensions Play? Front. Psychol. 2017, 8. [CrossRef]

42. Seaman, C.S.; Weber, R. Undisclosed flexibility in computing and reporting structural equation models in communication science. Commun. Met. Measur. 2015, 9, 208-232. [CrossRef] 
43. González-Cutre, D.; Sicilia, A.; Moreno, J.A. Cognitive-social model of achievement motivation in physical education. Psicothema 2008, 20, 642-651.

44. Schutte, N.S.; Malouff, J.M.; Hall, L.E.; Haggerty, D.J.; Cooper, J.T.; Golden, J.; Dornhein, L. Development and validation of a measure of emotional intelligence. J. Individ. Differ. 1998, 25, 167-177. [CrossRef]

45. García-Coll, V.; Graupera-Sanz, J.L.; Ruiz-Pérez, L.; Palomo-Nieto, M. Inteligencia emocional en el deporte: Emotional Inteligence in Sport: Validation of the Schutte Self Report Inventory (SSRI) in Spanish athletes. Cuad. Psicol. Dep. 2013, 13, 25-36. [CrossRef]

46. Spielberger, C.D.; Gorsuch, R.; Luschene, R. The State-Trait Anxiety Inventory; Psychologist Press: Palo Alto, CA, USA, 1970.

47. Ronquillo, H. Association between occlusal conditions, trait-state anxiety levels and temporomandibular disorders in a group of dental students. Cart Odontol. 2012, 1, 27-42.

48. Horikawa, M.; Yagi, A. The Relationships among Trait Anxiety, State Anxiety and the Goal Performance of Penalty Shoot-Out by University Soccer Players. PLoS ONE 2012, 7, e35727. [CrossRef]

49. Marsh, H.W. Handbook of Sport Psychology, 3rd ed.; Tenenbaum, G., Eklund, R.C., Eds.; John Wiley \& Sons Inc.: Hoboken, NJ, USA, 2007.

50. Hill, B.D.; Foster, J.D.; Sofko, C.; Elliott, E.M.; Shelton, J.T. The interaction of ability and motivation: Average working memory is required for need for cognition to positively benefit intelligence and the effect increases with ability. Personal. Individ. Differ 2016, 98, 225-228. [CrossRef]

51. Jones, L.; Karageorghis, C.I.; Lane, A.M.; Bishop, D.T. The influence of motivation and attentional style on affective, cognitive, and behavioral outcomes of an exercise class. Scan. J. Med. Scin. Sports 2017, 27, 124-135. [CrossRef]

52. Lee, Y.H.; Chelladurai, P. Affectivity, emotional labor, emotional exhaustion, and emotional intelligence in coaching. J. Appl. Sport Psychol. 2016, 28, 170-184. [CrossRef]

53. Stenling, A.; Ivarsson, A.; Hassmén, P.; Lindwall, M. Longitudinal associations between athletes' controlled motivation, ill-being, and perceptions of controlling coach behaviors: A Bayesian latent growth curve approach. Psychol. Sport Exerc. 2017, 30, 205-214. [CrossRef]

54. Zurita, F.; Zafra, E.; Valdivia, P.; Rodríguez, S.; Castro, M.; Muros, J.J. Analysis of resilience, self-concept and motivation in judo according to gender. Rev. Psicol. Dep. 2017, 26, 71-81.

55. Rottensteiner, C.; Tolvanen, A.; Laakso, L.; Konttinen, N. Youth athletes' motivation, perceived competence, and persistence in organized team sports. J. Sport Behav. 2015, 38, 432.

56. Zarauz-Sancho, A.; Ruiz-Juan, F. Determining factors of motivation in Spanish veteran athletes. Rev. Lat. Psicol. 2015, 47, 34-42. [CrossRef]

57. Hogue, C.M.; Fry, M.D.; Fry, A.C. The differential impact of motivational climate on adolescents' psychological and physiological stress responses. Psychol. Sport Exerc. 2017, 30, 118-127. [CrossRef]

58. Davies, M.J.; Babkes-Stellino, M.; Nichols, B.A.; Coleman, L.M. Other-initiated motivational climate and youth hockey players' good and poor sport behaviors. J. Appl. Sport Psychol. 2016, 28, 78-96. [CrossRef]

59. Domínguez-Escribano, M.; Ariza-Vargas, L.; Taberner, C. Motivational variables involved in commitment of female soccer players at different competitive levels. Soccer Soc. 2017, 18, 801-816. [CrossRef]

60. Matthews, W. Group Cohesion, Motivational Climate, and Collective Efficacy Beliefs in Community College and University Bands. J. Band Res. 2016, 51, 1-67. [CrossRef]

61. Møllerløkken, N.E.; Lorås, H.; Pedersen, A.V. A Comparison of Players' and Coaches' Perceptions of the Coach-Created Motivational Climate within Youth Soccer Teams. Front. Psychol. 2017, 8, 1-10. [CrossRef]

62. McLaren, C.D.; Newland, A.; Eys, M.; Newton, M. Peer-initiated motivational climate and group cohesion in youth sport. J. Appl. Sport Psychol. 2017, 29, 88-100. [CrossRef]

63. Gross, M.J.; Hall, R.; Bringer, J.D.; Cook, C.J.; Kilduff, L.P.; Shearer, D.A. Resonant frequency training in elite sport: A case study example. J. Sport Psychol. Act. 2017, 8, 173-183. [CrossRef]

64. Ekaitz, S.; Arribas-Galarrag, S.; Cecchini, J.A.; Luis-De-Cos, I.; Otaegi, O. Differences in goal orientation, self-determined motivation, emotional intelligence and satisfaction with sporting results among expert and novice canoeists. Cuad. Psicol. Dep. 2014, 14, 21-30. [CrossRef]

65. Ma, C.; Monsma, E. Testing Cross-Cultural Generalizability of the Task and Ego Orientation in Sport Questionnaire across American and Chinese Samples. PLoS ONE 2016, 11, e0158953. [CrossRef]

66. Yukhymenko-Lescroart, M.A. On identity and sport conduct of student-athletes: Considering athletic and academic contexts. Psychol. Sport Exerc. 2018, 34, 10-19. [CrossRef] 
67. Neil, R.; Hanton, S.; Mellalieu, S.D.; Fletcher, D. Competition stress and emotions in sport performers: The role of further appraisals. Psychol. Sport Exerc. 2011, 12, 460-470. [CrossRef]

68. Latinjak, A.T.; Hatzigeorgiadis, A.; Zourbanos, N. Goal-Directed and Spontaneous Self-Talk in Anger-and Anxiety-Eliciting Sport-Situations. J. Appl. Sport Psychol. 2017, 29, 150-166. [CrossRef]

69. Huéscar-Hernández, E.; López-Mora, C.; Cervelló-Gimeno, E. Relationship of Leadership Styles, Group Cohesion, Power Equipment and Performance in Non-Professional Soccer Players. Univ. Psychol. 2017, 16, 125-138. [CrossRef]

70. Smirl, J.D.; Dierijck, J.K.; Wright, A.D.; Bryk, K.; van Donkelaar, P. Effect of sub-concussive impacts sustained throughout a contact-sport season on quiet stance centre of pressure. Br. J. Sports Med. 2017, 51, A28-A29. [CrossRef]

71. Thornton, C.; Sheffield, D.; Baird, A. A longitudinal exploration of pain tolerance and participation in contact sports. Scan. J. Pain 2017, 16, 36-44. [CrossRef]

72. Giza, C.C.; Prins, M.L.; Hovda, D.A. It's not all fun and games: Sports, concussions, and neuroscience. Neuron 2017, 94, 1051-1055. [CrossRef]

73. Brown, D.J.; Fletcher, D. Effects of psychological and psychosocial interventions on sport performance: A meta-analysis. Sports Med. 2017, 47, 77-99. [CrossRef]

74. Meira, C.M.; Fairbrother, J.T. Ego-oriented Learners Show Advantage in Retention and Transfer of Balancing Skill. J. Motor Learn. Dev. 2017, 1-21. [CrossRef]

75. Morgan, K. Reconceptualizing motivational climate in physical education and sport coaching: An interdisciplinary perspective. Quest 2017, 69, 95-112. [CrossRef]

76. Ros, A.; Moya-Faz, F.; Garcés, F. Emotional intelligence and sport: Current state of research. Cuad. Psicol. Dep. 2013, 13, 105-112.

77. Williams, S.E.; van Zanten, J.J.; Trotman, G.P.; Quinton, M.L.; Ginty, A.T. Challenge and threat imagery manipulates heart rate and anxiety responses to stress. Int. J. Psychophysiol. 2017, 117, 111-118. [CrossRef]

(C) 2019 by the authors. Licensee MDPI, Basel, Switzerland. This article is an open access article distributed under the terms and conditions of the Creative Commons Attribution (CC BY) license (http://creativecommons.org/licenses/by/4.0/). 